\title{
Exploration of elastic scattering rates for supersymmetric dark matter
}

\author{
John Ellis \\ TH Division, CERN, Geneva, Switzerland \\ Andrew Ferstl \\ School of Physics and Astronomy, University of Minnesota, Minneapolis, Minnesota 55455 \\ Keith A. Olive \\ School of Physics and Astronomy, University of Minnesota, Minneapolis, Minnesota 55455 \\ and Theoretical Physics Institute, University of Minnesota, Minneapolis, Minnesota 55455
}

(Received 18 July 2000; published 23 February 2001)

\begin{abstract}
We explore the possible cross sections for the elastic scattering of neutralinos $\chi$ on nucleons $p, n$ in the minimal supersymmetric extension of the standard model (MSSM). Universality of the soft supersymmetrybreaking scalar masses for the Higgs multiplets is not assumed, but the MSSM parameters are nevertheless required to lead consistently to an electroweak vacuum. We explore systematically the region of MSSM parameter space where CERN LEP and other accelerator constraints are respected, and the relic neutralino density lies in the range $0.1 \leqslant \Omega_{\chi} h^{2} \leqslant 0.3$ preferred by cosmology. We also discuss models with $\Omega_{\chi} h^{2}<0.1$, in which case we scale the density of supersymmetric dark matter in our galactic halo by $\Omega_{\chi} h^{2} / 0.1$, allowing for the possible existence of some complementary form of cold dark matter. We restrict our attention to $\tan \beta$ $\leqslant 10$, for which reliable relic-density calculations are available. Within this range of $\tan \beta$, we find values of the cross sections that are considerably lower than the present experimental sensitivities. At low neutralino masses, $m_{\chi} \lesssim 100 \mathrm{GeV}$, the cross sections may be somewhat higher than in the constrained MSSM with universal soft Higgs boson masses, though they are generally lower. In the case of large $m_{\chi}$, the cross sections we find may be considerably larger than in the constrained model, but still well below the present experimental sensitivity.

DOI: 10.1103/PhysRevD.63.065016

PACS number(s): 14.80.Ly, 95.30.Cq, 95.35.+d
\end{abstract}

\section{INTRODUCTION}

One of the key issues at the frontier between particle physics and cosmology is the nature of the non-baryonic dark matter that apparently dominates the matter density of the Universe. This is probably dominated by cold dark matter, with a density that probably falls within the range 0.2 $<\Omega_{C D M}<0.5$ [1], and may be in the form of massive weakly interacting particles. It is therefore particularly important to search for such dark matter particles [2], and one of the most direct strategies is the search for relic particle scattering on nuclei in a laboratory detector [3]. Many experiments around the world are engaged in this search, largely motivated by the cross sections calculated assuming that the cold dark matter is dominated by the lightest neutralino $\chi[4]$ of the minimal supersymmetric extension of the standard model (MSSM) [5].

We recently re-evaluated [6] the spin-dependent and spinindependent cross sections for neutralino scattering on protons and neutrons for $\tan \beta \leqslant 10[7,8]$, assuming universality for all of the soft supersymmetry-breaking mass parameters of the MSSM including the Higgs multiplets, incorporating the latest available CERN $e^{+} e^{-}$collider LEP constraints on the MSSM parameter space, and assuming that the cosmological density of the relic neutralino falls within the range $0.1<\Omega_{\chi} h^{2}<0.3$, corresponding to the favored range of $\Omega_{C D M}$ and a Hubble expansion rate $0.6 \leqslant h \leqslant 0.8$ in units $H_{0} \equiv 100 \times h \mathrm{~km} / \mathrm{s} / \mathrm{Mpc}$. We used the latest information from chiral symmetry [9,10], low-energy $\pi-p, n$ scattering [11] and deep-inelastic lepton-nucleon scattering [12] to fix the hadronic matrix elements. Our calculations fell considerably below the present experimental sensitivities [13], as well as the highest theoretical estimates available in the literature [14], some of which used less restrictive assumptions. There are, however, some other recent lower estimates: see [15], for example, which is in good agreement with our previous work [6].

Shortly after our paper appeared, the DAMA Collaboration confirmed [16] their previous evidence for the annual modulation of energy deposits in their scintillation detector, which they interpret as due to the scattering of some cold dark matter particle with mass between about 50 and 100 $\mathrm{GeV}$, and spin-independent cross section on a proton between about $10^{-6}$ and $10^{-5} \mathrm{pb}$. This cross section range is considerably larger than we found previously [6], though consistent with the range allowed by some previous cross section estimates. Subsequent to the DAMA paper, the CDMS Collaboration has reported [17] negative results from their experiment, establishing an upper limit on the spinindependent cross section that excludes most, but not all, of the range suggested by DAMA.

This unresolved situation motivates us to explore more widely the possible neutralino-proton cross sections in the MSSM, including both the spin-dependent and spinindependent (scalar) contributions. As before, we impose the latest constraints on the MSSM parameter space imposed by the LEP and other experiments [18,19], such as measurements of $b \rightarrow s \gamma$ decay. We again restrict our attention to $\tan \beta \leqslant 10$, for which reliable relic-density calculations including coannihilation are available. It is important to note 
that the LEP limits we use here [19] have been updated significantly compared to what we used in [6]. Notably, the chargino and particularly the Higgs boson mass limits we use here are stronger. The latter has a substantial effect at $\tan \beta$ $=3$ : in addition to the consequent direct reduction in the Higgs-exchange contribution to the scalar cross section, the improved lower limit on the Higgs boson mass further restricts $m_{0}$ and $m_{1 / 2}$ from below, because of their contribution to $m_{h}$ via radiative corrections. Also, previously we did not use the $b \rightarrow s \gamma$ constraint, which we implement here by requiring $m_{A}>300 \mathrm{GeV}$ for $\mu<0$. All of these effects tend to remove some of the higher cross sections that we found previously, particularly at low $m_{\chi}$.

The main thrust of this paper, however, is to relax two of the theoretical assumptions made in our previous work.

The absence of large flavor-changing neutral interactions suggests that the soft supersymmetry-breaking scalar mass parameters $m_{0_{i}}$ of the MSSM may be universal for different quark and lepton flavors. However, there is no strong phenomenological or theoretical reason why the $m_{0_{i}}$ should be the same for the Higgs multiplets as for squarks and sleptons, and we relax this universality assumption in this work. It is known that, in this case, the lightest neutralino $\chi$ might be mainly a Higgsino, but this particular option is greatly restricted by LEP data $[18,19]$.

Neutralinos might not constitute all the cold dark matter, but might be complemented by other particles such as axions or superheavy relics. In this case, $\Omega_{\chi}<\Omega_{C D M}$, and $\Omega_{\chi} h^{2}$ $<0.1$ becomes a possibility. For any given neutralino mass, $\Omega_{\chi}$ may be decreased by increasing the $\chi$ annihilation cross sections, which is often correlated with an enhanced elastic $\chi$-proton scattering cross section. Before concluding that cold dark matter detection becomes easier in this case, however, one must consider what fraction of our galactic halo density $\rho_{\text {halo }}$ could be composed of neutralinos. Since the process of halo formation is essentially independent of the nature of the cold dark matter, as long as it is non-relativistic and weakly interacting, one should expect that

$$
\rho_{\chi}=\rho_{\text {halo }} \times\left(\frac{\Omega_{\chi}}{\Omega_{C D M}}\right) .
$$

In an effort to be as optimistic as is reasonable, we assume that $\rho_{\chi}=\rho_{\text {halo }}$ if $\Omega_{\chi} h^{2} \geqslant 0.1$, and rescale: $\rho_{\chi}$ $=\rho_{\text {halo }} \times\left(\Omega_{\chi} h^{2} / 0.1\right)$ if $\Omega_{\chi} h^{2} \leqslant 0.1$.

In our previous work [6], in which we assumed universality for the Higgs boson masses (UHM) at the conventional supersymmetric grand unified theory (GUT) scale $\sim 10^{16}$ $\mathrm{GeV}$, and the canonical range $0.1<\Omega_{\chi} h^{2}<0.3$, we found that the possible ranges of elastic scattering cross sections were very narrow for any fixed values of $m_{\chi}, \tan \beta \leqslant 10$ and the sign of $\mu$, even allowing for plausible uncertainties in the hadronic inputs [9-12], and that they were always orders of magnitude below the present sensitivities [13], even for the smallest allowed values of $m_{\chi} \sim 50 \mathrm{GeV}[18,19]$. Specifically, the maximum value we found for the spin-dependent $\chi-p$ elastic scattering cross section for $3 \leqslant \tan \beta \leqslant 10$ was well below $10^{-3} \mathrm{pb}$, attained for $m_{\chi} \sim 60 \mathrm{GeV}$, and the maxi- mum value we found for the spin-independent $\chi-p$ elastic scattering cross section for $3 \leqslant \tan \beta \leqslant 10$ was $\sim 10^{-7} \mathrm{pb}$, again attained for $m_{\chi} \sim 60 \mathrm{GeV}$. The corresponding experimental sensitivities are $\sim 1 \mathrm{pb}$ and $\sim 3 \times 10^{-6} \mathrm{pb}$, respectively. At higher neutralino masses, the predicted cross sections were significantly smaller still.

In the constrained version of the MSSM, when all soft scalar masses, including the Higgs boson masses, are set equal at the unification scale (UHM), there are four independent parameters, the soft scalar masses, $m_{0}$, the gaugino masses, $m_{1 / 2}$, the soft trilinear mass terms, $A$ (assumed to be universal), and $\tan \beta$. In addition, there is the freedom to choose the sign of the Higgs mixing mass $\mu$. Previously we scanned the $m_{0}-m_{1 / 2}$ parameter space for fixed $\tan \beta \leqslant 10$ and $\operatorname{sgn}(\mu)$. Our results were not very sensitive to $A$.

Now that we relax the universal Higgs-boson-mass assumption (UHM), we find much broader ranges of elastic scattering cross sections for any fixed values of $m_{\chi}, \tan \beta$ $\leqslant 10$ and the sign of $\mu$. As previously, we perform a systematic scan of the region of the $m_{0}, m_{1 / 2}$ parameter space of the MSSM that is consistent with accelerator constraints. Here, $m_{0}$ refers only to a common squark and slepton mass, and the two Higgs boson soft masses $m_{1}$ and $m_{2}$ are fixed by the conditions of electroweak symmetry breaking, since we allow $\mu$ and the Higgs pseudoscalar mass $m_{A}$ to be free parameters. Thus, we scan over $m_{0}, m_{1 / 2}, \mu, m_{A}$, and $A$ for fixed $\tan \beta$. The details of these scans are given below, where we document which parameter choices fail which LEP constraint and/or the cosmological relic density requirement.

We find that the elastic scattering cross sections may be somewhat larger than we found before in the UHM case, particularly for larger $m_{\chi}$. However, the absolute values are still well below the present experimental sensitivities [13], at least for the canonical range $0.1<\Omega_{\chi} h^{2}<0.3$ for the relic neutralino density. This remains true when we consider $\Omega_{\chi} h^{2}<0.1$, but rescale the halo density as described above.

We cannot exclude the possibility that there might be some variant of the MSSM that could accommodate the cold dark matter scattering interpretation of the DAMA data, but this would require an extension of the framework discussed here. One possibility might be to adopt a larger value of $\tan \beta$ [20]: we restrict our attention to $\tan \beta \leqslant 10$ to avoid uncertainties in the relic density calculations. To be complete, these should include coannihilation effects, which are not currently available. We plan to return in a future publication to the necessary careful calculation of these and renormalization-group evolution effects in the context of a more complete study of MSSM phenomenology at large $\tan \beta$.

Another possibility might be to relax further the universality assumptions for soft supersymmetry-breaking masses, either in the scalar or the gaugino sector. In particular, models in which $m_{\tilde{q}} / m_{\tilde{l}}$ is smaller than in the models discussed here might be able to accommodate larger elastic $\chi$-proton rates for any given value of $\Omega_{\chi}$. Another way to reduce $m_{\tilde{q}} / m_{\tilde{l}}$, with a similar effect, could be to postulate universality at a lower, intermediate renormalization scale, below the conventional supersymmetric GUT scale [21]. 


\section{THEORETICAL AND PHENOMENOLOGICAL BACKGROUND}

We review in this section relevant aspects of the MSSM [5]. The neutralino LSP is the lowest-mass eigenstate combination of the $B$-ino $\widetilde{B}, W$-ino $\widetilde{W}$ and Higgsinos $\widetilde{H}_{1,2}$, whose mass matrix $N$ is diagonalized by a matrix $Z$ : $\operatorname{diag}\left(m_{\chi_{1}, \ldots, 4}\right)=Z^{*} N Z^{-1}$. The composition of the lightest neutralino may be written as

$$
\chi=Z_{\chi_{1}} \widetilde{B}+Z_{\chi^{2}} \widetilde{W}+Z_{\chi^{3}} \widetilde{H}_{1}+Z_{\chi^{4}} \widetilde{H}_{2} .
$$

As previously, we neglect $C P$ violation in this paper, so that there are no $C P$-violating phases in the neutralino mass matrix and mixing. For the effects of $C P$-violating phases on the neutralino scattering cross section see [22-25]. We assume universality at the supersymmetric GUT scale for the $U(1)$ and $S U(2)$ gaugino masses: $M_{1,2}=m_{1 / 2}$, so that $M_{1}$ $=\frac{5}{3} \tan ^{2} \theta_{W} M_{2}$ at the electroweak scale.

We also assume GUT-scale universality for the soft supersymmetry-breaking scalar masses $m_{0}$ of the squarks and sleptons, but not for the Higgs bosons, in contrast to [6]. We further assume GUT-scale universality for the soft supersymmetry-breaking trilinear terms $A$. Our treatment of the sfermion mass matrices $M$ follows those in [22], and we refer the interested reader to [6] for further details and notation. It suffices here to recall that, $C P$ being conserved, the sfermion mass-squared matrix for each flavor $f$ is diagonalized by a rotation through an angle $\theta_{f}$. We treat as free parameters $m_{1 / 2}$ (we actually use $M_{2}$ which is equal to $m_{1 / 2}$ at the unification scale), the soft supersymmetry-breaking scalar mass scale $m_{0}$ (which in the present context refers only to the universal sfermion masses at the unification scale), $A$ and $\tan \beta$. In addition, we treat $\mu$ and the pseudoscalar Higgs boson mass $m_{A}$ as independent parameters, and thus the two Higgs boson soft masses $m_{1}$ and $m_{2}$, are specified by the electroweak vacuum conditions, which we calculate using $m_{t}=175 \mathrm{GeV}$. $^{1}$

The MSSM Lagrangian leads to the following low-energy effective four-fermion Lagrangian suitable for describing elastic $\chi$-nucleon scattering [22]:

$$
\begin{aligned}
\mathcal{L}= & \bar{\chi} \gamma^{\mu} \gamma^{5} \chi \overline{q_{i}} \gamma_{\mu}\left(\alpha_{1 i}+\alpha_{2 i} \gamma^{5}\right) q_{i}+\alpha_{3 i} \bar{\chi} \chi \overline{q_{i}} q_{i} \\
& +\alpha_{4 i} \bar{\chi} \gamma^{5} \chi \overline{q_{i}} \gamma^{5} q_{i}+\alpha_{5 i} \bar{\chi} \chi \overline{q_{i}} \gamma^{5} q_{i}+\alpha_{6 i} \bar{\chi} \gamma^{5} \chi \overline{q_{i}} q_{i} .
\end{aligned}
$$

This Lagrangian is to be summed over the quark generations, and the subscript $i$ labels up-type quarks $(i=1)$ and downtype quarks $(i=2)$. The terms with coefficients $\alpha_{1 i}, \alpha_{4 i}, \alpha_{5 i}$ and $\alpha_{6 i}$ make contributions to the elastic scattering cross section that are velocity-dependent, and may be neglected for our purposes. In fact, if the $C P$-violating phases are absent as assumed here, $\alpha_{5}=\alpha_{6}=0[23,24]$. The coefficients relevant for our discussion are

\footnotetext{
${ }^{1} \mathrm{We}$ have checked that varying $m_{t}$ by $\pm 5 \mathrm{GeV}$ has a negligible effect on our results.
}

$$
\begin{aligned}
\alpha_{2 i}= & \frac{1}{4\left(m_{1 i}^{2}-m_{\chi}^{2}\right)}\left[\left|Y_{i}\right|^{2}+\left|X_{i}\right|^{2}\right]+\frac{1}{4\left(m_{2 i}^{2}-m_{\chi}^{2}\right)} \\
& \times\left[\left|V_{i}\right|^{2}+\left|W_{i}\right|^{2}\right]-\frac{g^{2}}{4 m_{Z}^{2} \cos ^{2} \theta_{W}}\left[\left|Z_{\chi_{3}}\right|^{2}-\left|Z_{\chi_{4}}\right|^{2}\right] \frac{T_{3 i}}{2}
\end{aligned}
$$

and

$$
\begin{aligned}
\alpha_{3 i}= & -\frac{1}{2\left(m_{1 i}^{2}-m_{\chi}^{2}\right)} \operatorname{Re}\left[\left(X_{i}\right)\left(Y_{i}\right)^{*}\right]-\frac{1}{2\left(m_{2 i}^{2}-m_{\chi}^{2}\right)} \\
& \times \operatorname{Re}\left[\left(W_{i}\right)\left(V_{i}\right)^{*}\right]-\frac{g m_{q i}}{4 m_{W} B_{i}}\left[\operatorname{Re}\left(\delta_{1 i}\left[g Z_{\chi^{2}}-g^{\prime} Z_{\chi 1}\right]\right)\right. \\
& \times D_{i} C_{i}\left(-\frac{1}{m_{H_{1}}^{2}}+\frac{1}{m_{H_{2}}^{2}}\right)+\operatorname{Re}\left(\delta_{2 i}\left[g Z_{\chi^{2}}-g^{\prime} Z_{\chi 1}\right]\right) \\
& \left.\times\left(\frac{D_{i}^{2}}{m_{H_{2}}^{2}}+\frac{C_{i}^{2}}{m_{H_{1}}^{2}}\right)\right]
\end{aligned}
$$

where

$$
\begin{aligned}
X_{i} & \equiv \eta_{11}^{*} \frac{g m_{q_{i}} Z_{\chi^{5}-i}^{*}}{2 m_{W} B_{i}}-\eta_{12}^{*} e_{i} g^{\prime} Z_{\chi^{1}}^{*} \\
Y_{i} & \equiv \eta_{11}^{*}\left(\frac{y_{i}}{2} g^{\prime} Z_{\chi 1}+g T_{3 i} Z_{\chi 2}\right)+\eta_{12}^{*} \frac{g m_{q_{i}} Z_{\chi 5-i}}{2 m_{W} B_{i}} \\
W_{i} & \equiv \eta_{21}^{*} \frac{g m_{q_{i}} Z_{\chi 5}^{*}}{2 m_{W} B_{i}} \\
V_{i} & \equiv \eta_{22}^{*} \frac{g m_{q_{i}} Z_{\chi 5-i}}{2 m_{W} B_{i}}+\eta_{21}^{*}\left(\frac{y_{i}}{2} g^{\prime} Z_{\chi 1}+g T_{3 i} Z_{\chi 2}\right)
\end{aligned}
$$

where $y_{i}, T_{3 i}$ denote hypercharge and isospin, and

$$
\begin{aligned}
& \delta_{1 i}=Z_{\chi^{3}}\left(Z_{\chi^{4}}\right), \quad \delta_{2 i}=Z_{\chi^{4}}\left(-Z_{\chi^{3}}\right), \\
& B_{i}=\sin \beta(\cos \beta), \quad A_{i}=\cos \beta(-\sin \beta), \\
& C_{i}=\sin \alpha(\cos \alpha), \quad D_{i}=\cos \alpha(-\sin \alpha)
\end{aligned}
$$

for up (down) type quarks. We denote by $m_{H_{2}}<m_{H_{1}}$ the two scalar Higgs boson masses, and $\alpha$ denotes the Higgs mixing angle. $^{2}$

As discussed in [6], the elastic cross section for scattering off a nucleus can be decomposed into a scalar (spinindependent) part obtained from the $\alpha_{2 i}$ term in Eq. (3), and

\footnotetext{
${ }^{2}$ We note that Eq. (5) is taken from [23] and that Eqs. (4),(5) agree with $[2,7,24]$.
} 
a spin-dependent part obtained from the $\alpha_{3 i}$ term. Each of these can be written in terms of the cross sections for elastic scattering for scattering off individual nucleons. We reevaluated the relevant matrix elements in [6]. Here we limit ourselves to recalling that:

There are uncertainties in the scalar part of the cross section associated with the ratios of the light-quark masses, which we take from [9]:

$$
\frac{m_{u}}{m_{d}}=0.553 \pm 0.043, \quad \frac{m_{s}}{m_{d}}=18.9 \pm 0.8
$$

and information from chiral symmetry applied to baryons. Here the principal uncertainty is associated with the experimental value of the $\pi$-nucleon $\sigma$ term and the corresponding values of the ratios of the $B_{q} \equiv\langle p|\bar{q} q| p\rangle$. Following [10], we use

$$
z \equiv \frac{B_{u}-B_{s}}{B_{d}-B_{s}}=1.49
$$

with a negligible experimental error, and [11]

$$
y \equiv \frac{2 B_{s}}{B_{d}+B_{u}}=0.2 \pm 0.1
$$

which yields

$$
\frac{B_{d}}{B_{u}}=0.73 \pm 0.02 .
$$

The difference between the scalar parts of the cross sections for scattering off protons and neutrons are rather small.

The spin-dependent part of the elastic $\chi$-nucleus cross section can be written in terms of axial-current matrix elements $\Delta_{i}^{(p, n)}$ that parametrize the quark spin content of the nucleon. We extract from a recent global analysis [12] the values

$$
\begin{aligned}
& \Delta_{u}^{(p)}=0.78 \pm 0.04, \quad \Delta_{d}^{(p)}=-0.48 \pm 0.04, \\
& \Delta_{s}^{(p)}=-0.15 \pm 0.04
\end{aligned}
$$

where the errors are essentially $100 \%$ correlated for the three quark flavors. In the case of the neutron, we have $\Delta_{u}^{(n)}$ $=\Delta_{d}^{(p)}, \Delta_{d}^{(n)}=\Delta_{u}^{(p)}$, and $\Delta_{s}^{(n)}=\Delta_{s}^{(p)}$.

\section{COSMOLOGICAL AND EXPERIMENTAL CONSTRAINTS}

As noted in [6], several convergent measures of cosmological parameters [1] have suggested that the cold dark matter density $\Omega_{C D M}=0.3 \pm 0.1$ and that the Hubble expansion rate $H \equiv h \times 100 \mathrm{~km} / \mathrm{s} / \mathrm{Mpc}: h=0.7 \pm 0.1$, leading to our preferred range $0.1 \leqslant \Omega_{C D M} h^{2} \leqslant 0.3$. The recent data on the spectrum of cosmic microwave background fluctuations from BOOMERANG [26] and MAXIMA [27] are consistent with this range, but do not significantly constrain it further.
The upper limit on $\Omega_{C D M}$ can be translated directly into the corresponding upper limit on $\Omega_{\chi}$. However, it is possible that there is more than one component in the cold dark matter, for example axions and/or superheavy relics as well as the lightest supersymmetric particle (LSP) $\chi$, opening up the possibility that $\Omega_{\chi}<0.1$. For a given value of $m_{\chi}$, values of the MSSM parameters which lead to $\Omega_{\chi}<0.1$ tend to have larger $\chi$ annihilation cross sections, and hence larger elastic scattering cross sections. Note, however, that the upper bound, $\Omega_{\chi} h^{2}<0.3$, is a firm upper bound relying only on the lower limit to the age of the Universe, $t_{U}>1.2 \times 10^{10}$ years (with $\Omega_{\text {total }} \leqslant 1$ ).

However, in such a "shared" cold dark matter scenario, the packing fraction of neutralinos in the galactic halo must be reduced. As discussed in [28], for example, dark matter particles are taken into the halo in "sheets" in phase space, whose thicknesses are determined by their initial (thermal) velocity. The "sheets" of cold dark matter particles are of negligible thickness, so the ratios of their densities in the halo are identical with their cosmological densities, and therefore

$$
\frac{\rho_{\chi}}{\rho_{C D M}}=\frac{\Omega_{\chi}}{\Omega_{C D M}} .
$$

On the other hand, the "sheets" of hot dark matter particles are of finite thickness related to their thermal velocities at the onset of structure formation, which limits the possible phasespace density of hot dark matter particles, so that $\rho_{H D M} / \rho_{C D M}<\Omega_{H D M} / \Omega_{C D M}$ in general [28]. Moreover, a large ratio $\Omega_{H D M} / \Omega_{C D M}$ is currently not expected.

The LSP detection rate also must be reduced correspondingly to Eq. (13). Accordingly, when we consider MSSM parameter choices that have $\Omega_{\chi} h^{2} \leqslant 0.1$, we rescale the calculated scattering rate by a factor $\Omega_{\chi} h^{2} / 0.1$. This rescaling by the minimal acceptable value of $\Omega_{C D M} h^{2}$ is relatively optimistic.

For the calculation of the relic LSP density, we have included radiative corrections [18] to the neutralino mass matrix and include all possible annihilation channels [29]. In the MSSM, it is well known that there are large regions of the $M_{2}, \mu$ parameter plane for which the LSP and the next lightest neutralino (NLSP) and/or chargino are nearly degenerate, namely in the Higgsino portion of the plane when $M_{2} \gg \mu$. It was shown $[30,31]$ that, in these regions, coannihilations between the LSP, NLSP, and charginos are of particular importance in determining the final relic density of LSPs, and these have been included in the present calculation. Inclusion of these coannihilation channels has the important consequence that, in the Higgsino regions where one expects larger elastic scattering cross sections, the relic abundance is substantially reduced. On the other hand, we do not include here coannihilations between the LSP and the sleptons $\widetilde{l}$ [32], in particular the lighter stau $\tilde{\tau}_{1}$, which were shown to play an important role in models with scalar mass universality also for 
the Higgs multiplets (UHM). ${ }^{3}$ These are known, in particular, to be important for determining the maximum possible generic value of $m_{\chi}$ in the UHM case, but are of less generic importance than $\chi-\chi^{\prime}-\chi^{ \pm}$coannihilations in the nonuniversal (NUHM) case considered here. For the same reason, we have also not implemented $\chi-\tilde{t}$ coannihilation [33]. The neglect of such $\chi-\widetilde{f}$ coannihilation processes is generally conservative as far as the elastic scattering rates are concerned, since any reduction they cause in $\Omega_{\chi} h^{2}$ is unlikely to be compensated by a corresponding enhancement in the elastic scattering cross section. We also do not pay any particular attention to the narrow parameter slice of mixed gaugino or Higgsino dark matter where $|\mu| \propto m_{1 / 2}$ and $m_{\chi}$ may become large [34], because this requires an adjustment of parameters at the $\%$ level, and is hence not generic. However, these are sampled, with the appropriate weighting, in our general randomized scan of the parameter space.

The lower limit on $m_{\chi}$ depends on the sparticle search limits provided by LEP and other experiments $[18,19]$. The most essential of these for our current purposes are those provided by the experimental lower limits on the lighter chargino mass $m_{\chi^{ \pm}}$and the lighter scalar Higgs boson mass $m_{H_{2}}$. As discussed in [19], here we assume a lower limit $m_{\chi^{ \pm}} \geqslant 101 \mathrm{GeV}$. The impact of the recently-improved lower limit on the Higgs boson mass [35] is potentially more significant [19], particularly for $\tan \beta=3$, as displayed in Figs. 6 of [19]. The present experimental lower limit for $\tan \beta=3$ approaches $m_{\mathrm{H}_{2}}>107 \mathrm{GeV}$ [35]. In implementing this constraint, we allow a safety margin of $\sim 3 \mathrm{GeV}$ in the MSSM calculations of $m_{H_{2}}$ [36], and hence require the MSSM calculation to yield $m_{H_{2}}>104 \mathrm{GeV}$ for $\tan \beta=3$. In the case of $\tan \beta=10$, the LEP constraint on the MSSM Higgs boson mass is weaker (see Fig. 6 of [19]), and we require only $m_{H_{2}}>86 \mathrm{GeV}$, which includes again a $3 \mathrm{GeV}$ margin of uncertainty. The corresponding limit on $m_{0}$ and $m_{1 / 2}$ in this case may be ignored [19]. The other two constraints that we implement are on sfermion masses, which we require to be (i) larger than $92 \mathrm{GeV}$, and (ii) larger than that of the lightest neutralino. We recall also the importance of the $b \rightarrow s \gamma$ constraint [37], which we implement in an approximate way, by requiring $m_{A}>300 \mathrm{GeV}$ for $\mu<0$ [19]. As also discussed in [19], requiring our present electroweak vacuum to be stable against transitions to a lower-energy state in which electromagnetic charge and color are broken (CCB) [38] would remove a large part of the cosmologically favored domain of MSSM parameter space. We have not implemented this optional requirement in the present study. In the next section, we will show the effect of the various experimental constraints on our scan of the parameter plane.

\section{MSSM PARAMETER SCAN}

We have scanned systematically the MSSM parameter space, taking into account the cosmological and experimen-

\footnotetext{
${ }^{3}$ Since we consider that complete relic density calculations including these coannihilation effects are not available for large $\tan \beta$, we restrict our attention to $\tan \beta \leqslant 10$.
}

tal constraints enumerated in the previous section and implementing the MSSM vacuum conditions for the representative choices $\tan \beta=3$ and 10. As discussed in [19], lower values of $\tan \beta$ are almost entirely excluded by LEP. Our parameter scan was over the following ranges of parameters:

$$
\begin{aligned}
0 & <m_{0}<1000, \\
80 & <|\mu|<2000, \\
80 & <M_{2}<1000, \\
0 & <m_{A}<1000, \\
-1000 & <A<1000 .
\end{aligned}
$$

The main scan, which covers $m_{0}, \mu$ and $M_{2}>100 \mathrm{GeV}$ and $m_{A}>300 \mathrm{GeV}$, was supplemented with smaller but significant subscans, to cover the smaller values of these four parameters as described below. The values of $m_{0}$ we use are fixed at the unification scale $\sim 10^{16} \mathrm{GeV}$, while the values of the remaining parameters, $\mu, M_{2}, m_{A}$, and $A$ are evaluated at the electroweak scale. The lower cut off on both $M_{2}$ and $\mu$ is due to the lower limit on the chargino mass.

As indicated above, we impose a lower limit $m_{A}>300$ $\mathrm{GeV}$ for $\mu<0$ to avoid problems with $b \rightarrow s \gamma$. As can be seen from Fig. 4 of [19], there are regions of $\mu<0$ which are also excluded by $b \rightarrow s \gamma$ when $m_{A}>300 \mathrm{GeV}$. Similarly, even for $\mu>0$, where we impose no cut off on $m_{A}$, we have included some points which should be excluded on the basis of $b \rightarrow s \gamma$ [19]. Therefore, our crude treatment of the $b$ $\rightarrow s \gamma$ constraint is quite conservative. A more sophisticated treatment would thin out the population of points in the range of cross sections that we find, not expand it. ${ }^{4}$

As can be seen in Table I, the overall scan was divided into three (four) specific regions for each value of $\tan \beta$ and $\mu$ negative (positive), each with the number of points listed. For each scan, we used a linear measure, which is reflected in the densities of points in the subsequent figures. The subscans with lower thresholds were designed to scour carefully the regions of MSSM parameter space close to the LEP exclusions, with the aim of ensuring that we sampled points close to their boundaries. For each subscan, we show the number of points which survive all the LEP experimental constraints discussed above, and we see that lower fractions of the low-threshold subscans survive them, in particular because they tend to yield excluded values of the chargino mass. Figure 1 provides some insight into the impacts of the different LEP constraints for the case $\tan \beta=3$ and $\mu>0$. We plot in Fig. 1 the points scanned in the $M_{2}-\mu$ parameter plane. In making this scatter plot, we show a randomly chosen subset of 5000 of the 90000 points sampled, ${ }^{5}$ since it is

\footnotetext{
${ }^{4}$ The existing theoretical calculations of $b \rightarrow s \gamma$ are not accurate at large $\tan \beta$ (private communication from P. Gambino, via G. Ganis), which is another reason why we do not yet study this case.

${ }^{5} \mathrm{We}$ have checked that there is no qualitative difference between this plot and the much denser plot with all points shown.
} 
TABLE I. Details of MSSM parameter scans, including the numbers of points that survive the LEP constraints and have a relic density in the favored range.

\begin{tabular}{|c|c|c|c|}
\hline Scan & Total points & Survived LEP & $0.1 \leqslant \Omega h^{2} \leqslant 0.3$ \\
\hline \multicolumn{4}{|c|}{$M_{2}, \mu, m_{0} \geqslant 100, m_{A} \geqslant 300$} \\
\hline $\tan \beta=3, \mu>0$ & 30000 & 17817 & 1552 \\
\hline $\tan \beta=3, \quad \mu<0$ & 30000 & 17210 & 901 \\
\hline $\tan \beta=10, \mu>0$ & 30000 & 26498 & 2588 \\
\hline $\tan \beta=10, \quad \mu<0$ & 30000 & 26507 & 2337 \\
\hline \multicolumn{4}{|c|}{$100 \geqslant M_{2}, \mu \geqslant 80, m_{0} \geqslant 100, m_{A} \geqslant 300$} \\
\hline $\tan \beta=3, \mu>0$ & 20000 & 75 & 0 \\
\hline $\tan \beta=3, \mu<0$ & 20000 & 4410 & 30 \\
\hline $\tan \beta=10, \mu>0$ & 20000 & 1632 & 14 \\
\hline $\tan \beta=10, \quad \mu<0$ & 20000 & 4480 & 58 \\
\hline \multicolumn{4}{|c|}{$M_{2}, \mu \geqslant 80, m_{0} \leqslant 100, m_{A} \geqslant 300$} \\
\hline $\tan \beta=3, \mu>0$ & 20000 & 2669 & 663 \\
\hline $\tan \beta=3, \quad \mu<0$ & 20000 & 2247 & 487 \\
\hline $\tan \beta=10, \mu>0$ & 20000 & 5394 & 2436 \\
\hline $\tan \beta=10, \quad \mu<0$ & 20000 & 5140 & 2377 \\
\hline \multicolumn{4}{|c|}{$M_{2}, \mu \geqslant 80, m_{0} \geqslant 0, m_{A} \leqslant 300$} \\
\hline $\tan \beta=3, \mu>0$ & 20000 & 2208 & 164 \\
\hline $\tan \beta=10, \mu>0$ & 20000 & 12096 & 1170 \\
\hline
\end{tabular}

much easier to pick out the relevant physical effects of the cuts in such a subset of points, the full plot being extremely dense.

We see that the chargino cut removes points at low values of $\mu$ and $M_{2}$, denoted by (green) pluses, that the Higgs cut then removes many more points with low $M_{2}$, denoted by (red) crosses, that the sfermion cut removes still more points with low $M_{2}$, denoted by (violet) triangles (this occurs at high $A$ and/or $\mu$ when there is a sizable off-diagonal component in the sfermion mass matrix), and that the LSP cut tends to remove points at higher $M_{2}$ denoted by (golden) diamonds. The surviving (blue) squares are spread over the $\mu, M_{2}$ plane, except for small values. Note that some points may fail to survive more than one of the above cuts. These are only denoted by the first cut tested and failed in the order listed above. The scans for the opposite sign of $\mu$ and for $\tan \beta=10$ exhibit similar features, and are omitted here. The only noticeable difference when $\mu>0$ is that not so many were points eliminated by the Higgs cut at large values of $M_{2}$ for $\mu<0$, because we imposed the limit $m_{A}>300 \mathrm{GeV}$ : for $\mu>0$, many more points were run with low $m_{A}$ and hence low $m_{H_{2}}$. Also, for $\tan \beta=10$, more points survive at low $\mu$ and/or $M_{2}$ due to the relaxed constraint on the Higgs boson mass.

The last column of Table I shows how many of the points that survive the LEP constraints and have relic densities in the cosmologically preferred range $0.1 \leqslant \Omega_{\chi} h^{2} \leqslant 0.3$. It is apparent that most of the preferred points emerge from the first scan with $M_{2}, \mu>100$, as the lower values which were explored thoroughly in the second subscan generally failed the chargino cut. More details of the scan over cosmological relic densities for $\tan \beta=3$ and $\mu>0$ are shown in Fig. 2. As in Fig. 1, we show only a randomly selected subset of 5000 points out of the total of approximately 22000 points which survived the LEP cuts. We see that, among the points that survived the previous LEP constraints, those with a small ratio of $\mu / M_{2}$ generally have too small a relic density, denoted by (green) pluses, as a result of over-efficient $\chi-\chi^{\prime}$ $-\chi^{ \pm}$coannihilation, whereas points with $\mu / M_{2} \sim 1$ to 5 tend to have too large a relic density, denoted by (red) crosses, particularly if $\mu$ and $M_{2}$ are individually large. The points with a relic density in the preferred range 0.1 $\leqslant \Omega_{\chi} h^{2} \leqslant 0.3$, denoted by (blue) squares, tend to accumulate around $\mu / M_{2} \sim 1 / 2$ or low $M_{2}$. The former points are in the transition region between over-efficient $\chi-\chi^{\prime}-\chi^{ \pm}$coannihilation and under-efficient annihilation at large $\mu$ and $M_{2}$, whereas the latter are in the region of low $M_{2}$ where careful implementation of the LEP constraints is essential. However, it is apparent from Fig. 2 that there are exceptions to these general trends. We do not discuss them in detail, but remark that we have made an attempt to understand at least those exceptions that lead to "unusual" elastic scattering cross sections.

\section{ELASTIC SCATTERING CROSS SECTIONS}

We now discuss the values of the elastic scattering cross sections that are attainable, bearing in mind the LEP and cosmological relic density constraints. Figure 3 illustrates the allowed ranges of elastic scattering cross sections for the points included in our scan for the particular case $\tan \beta$ $=3, \mu>0$, as it was described in the previous section. Plotted is a subset of 3000 of the 90000 points scanned, indicating which points survive all the LEP cuts, and which other points fail which LEP cut. We find similar results for $\tan \beta$ $=10 \mathrm{and} /$ or the opposite sign of $\mu$, with the exception that when $\mu<0$ we find some points trickling down below the apparent boundary at $\sim 10^{-10} \mathrm{pb}$ in Fig. 3(b), because of 


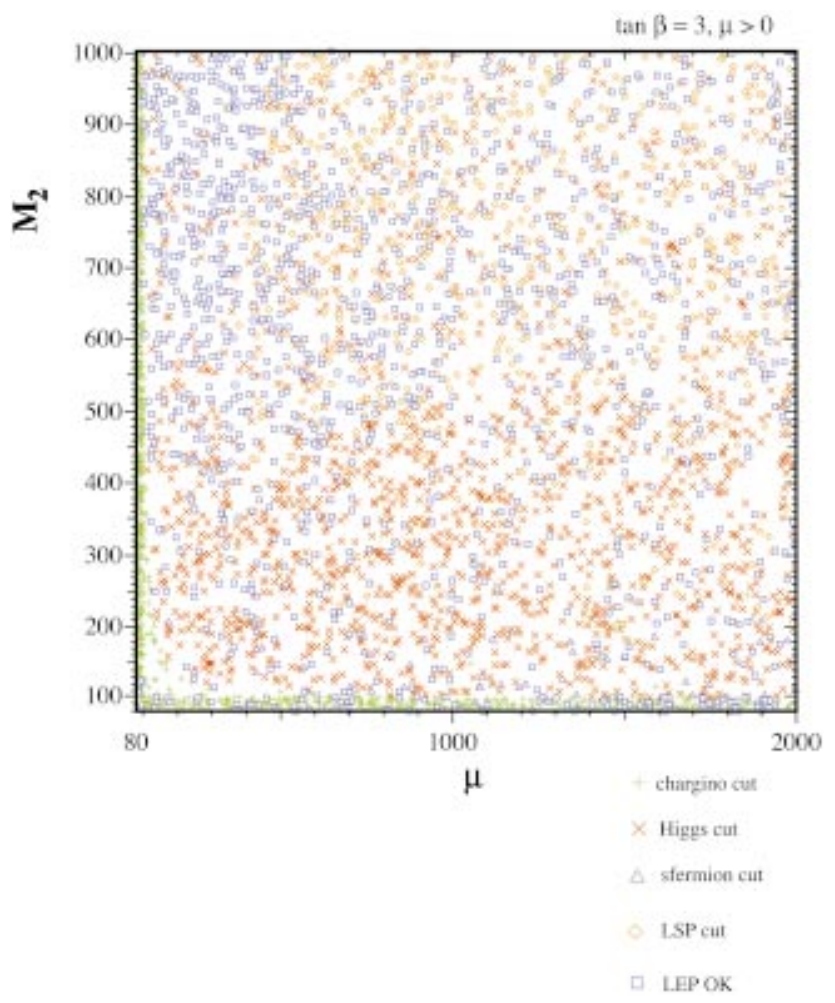

FIG. 1. (Color). Results of the scan of MSSM parameter space for $\tan \beta=3$ and $\mu>0$ summarized in Table I, illustrating the impacts of the various LEP constraints. We denote by (green) pluses the points that fail the chargino cut: $m_{\chi^{ \pm}} \geqslant 101 \mathrm{GeV}$, by (red) crosses the remaining points that survive the chargino cut but fail the Higgs cut: $m_{H_{2}} \geqslant 104 \mathrm{GeV}$, by (violet) triangles the points surviving the previous cuts that fail the sfermion cut: $m_{\tilde{f}} \geqslant 92 \mathrm{GeV}$, and by (golden) diamonds the points surviving the previous cuts that do not have the lightest neutralino as the LSP. The (blue) squares denote scan points that survive all these LEP cuts.

cancellations similar to those discussed in [6].

We note, in particular, that the LEP chargino and Higgs cuts remove many points with low $m_{\chi}$ and/or large elastic scattering cross sections. The sfermion mass cut is less important. The constraint that $\chi$ be the LSP removes quite a large number of points, populated more or less evenly in these cross section plots. The somewhat sparse set of points with very small cross sections give some measure of how low the cross section may fall in some special cases. These reflect instances where particular cancellations take place, examples of which were discussed in [6], and should not be regarded as generic. The lower boundary of the densely occupied region in Fig. 3 offers an answer to the question how low the elastic scattering cross sections may reasonably fall, roughly $\sigma \sim 10^{-9} \mathrm{pb}$ for the spin-dependent cross section and $\sim 10^{-10} \mathrm{pb}$ for the spin-independent cross section.

We would like to draw particular attention to the spin-independent cross section shown in Fig. 3(b). Notice that there are parameter choices with very large scattering cross sections. In this random selection, the cross-section may be as high as a few $\times 10^{-4} \mathrm{pb}$, and could even be larger than that claimed by DAMA. Indeed, in the full set of 90000

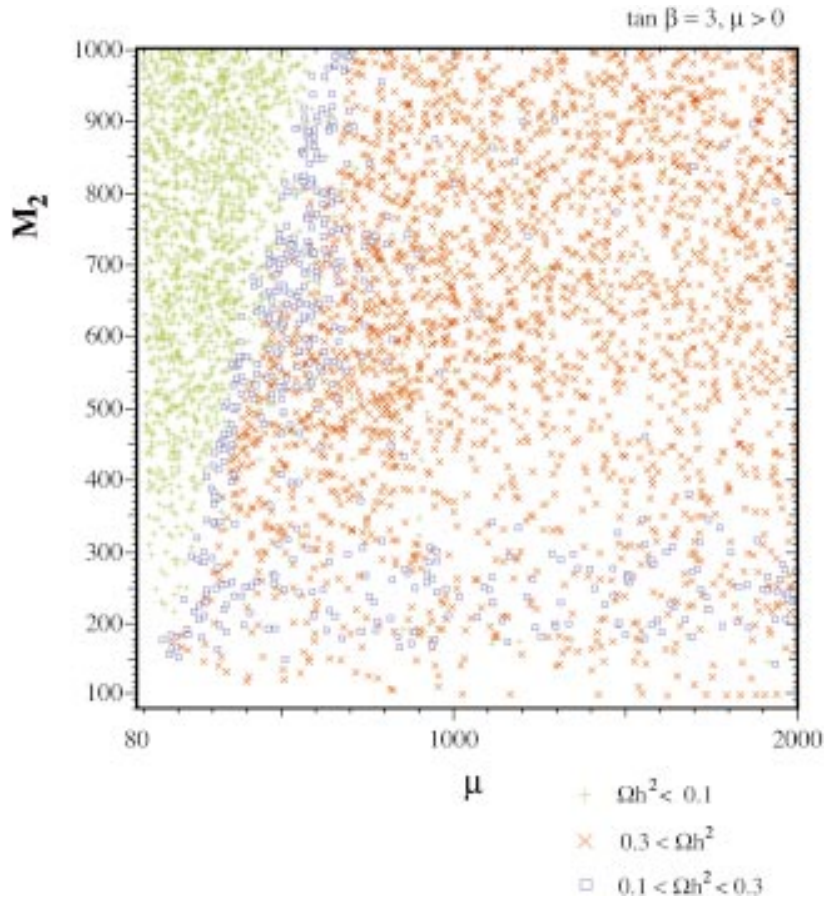

FIG. 2. (Color). Results of the scan of MSSM parameter space for $\tan \beta=3$ and $\mu>0$ summarized in Table I, illustrating the impact of the cosmological relic density constraint on the points that survived the LEP constraints illustrated in Fig. 1. We denote by (green) pluses the points that have too small a relic density: $\Omega_{\chi} h^{2}$ $<0.1$, by (red) crosses the points that have too high a relic density: $\Omega_{\chi} h^{2}>0.3$, and by (blue) squares the good points for which 0.1 $\leqslant \Omega_{\chi} h^{2} \leqslant 0.3$.

points scanned, there are even a few points which surpass $10^{-3} \mathrm{pb}$. However, all of these points have been excluded by LEP (primarily by the Higgs boson mass cut). The largest surviving cross section is slightly over $10^{-7} \mathrm{pb}$, in both the randomly selected subset and the full scan. For $\mu<0$, the upper boundary in the scalar cross section is about an order of magnitude lower, as was the case in the model with universal Higgs boson masses [6]. Note also that, for $\mu<0$, the limit $m_{A}>300 \mathrm{GeV}$ we impose removes the points with large cross sections (in this case with $\sigma_{\text {scalar }}$ $\geq 10^{-8} \mathrm{pb}$ ).

The next step is to implement the cosmological relic density constraints. We show in Fig. 4 the cross sections obtained for a representative subsample of points with $\tan \beta$ $=3, \mu>0$ that survive the LEP cuts, sorted according to the calculated values of $\Omega_{\chi} h^{2}$. Spin-dependent cross sections are plotted in panels (a) and (c), and spin-independent cross sections are plotted in panels (b) and (d). We include in panels (a) and (b) the cross sections calculated for unrealistic models with $\Omega_{\chi} h^{2}>0.3$, and without making any rescaling correction for points with $\Omega_{\chi} h^{2}<0.1$. The over-dense points with $\Omega_{\chi} h^{2}>0.3$, denoted by (red) crosses, have been removed in panels (c) and (d), and the cross sections for underdense points with $\Omega_{\chi} h^{2}<0.1$, denoted by (green) pluses, have been rescaled by the appropriate halo density fraction (1). As could be expected, the over-dense points tend to have 

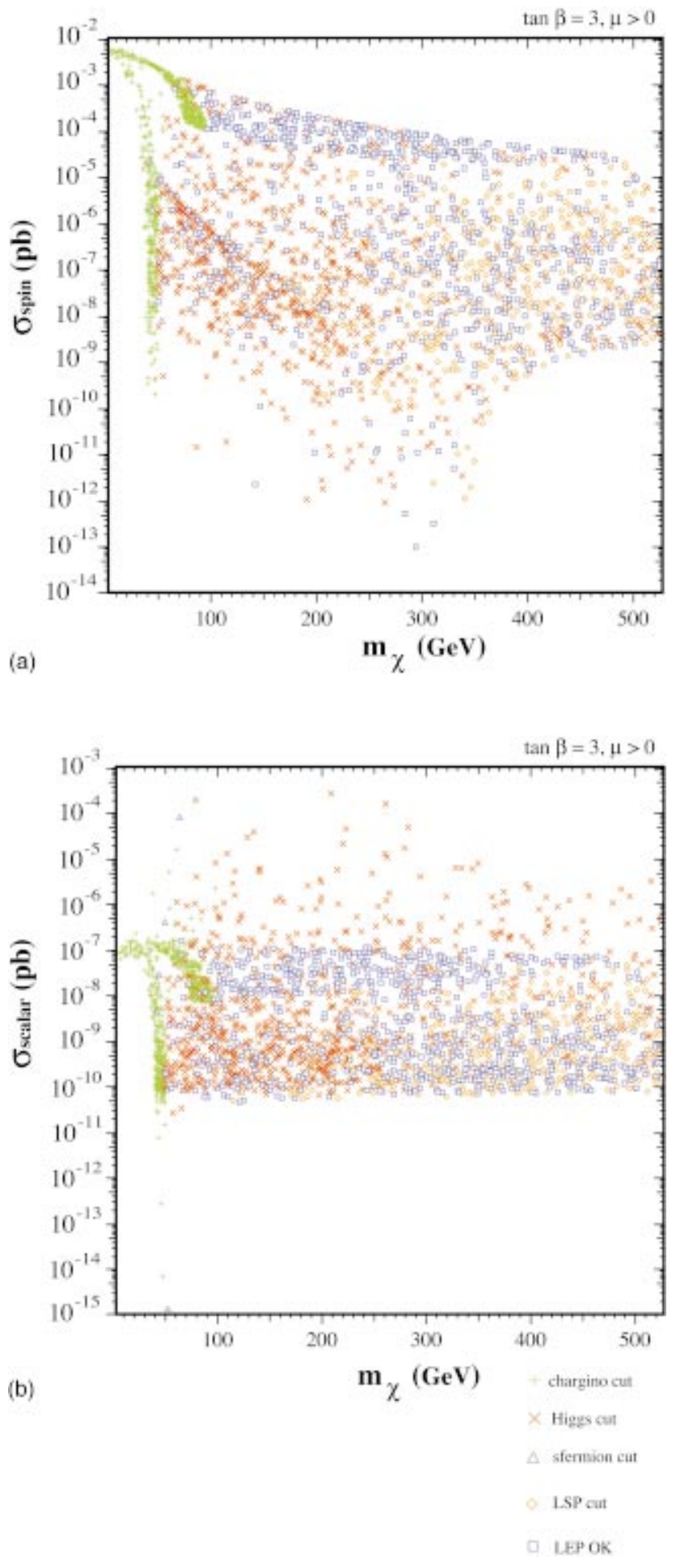

FIG. 3. (Color). Scatter plots of (a) the spin-dependent and (b) the spinindependent elastic scattering cross sections for $\tan \beta=3, \mu>0$ for a representative subsample of 3000 points, illustrating the impacts of the LEP constraints. The (green) plus signs denote points that fail the chargino mass constraint, which usually have small $m_{\chi}$ and sometimes large cross sections. The (red) crosses denote surviving points that fail the Higgs boson mass constraint, some of which have large spin-independent cross sections. The (violet) triangles denote surviving points that fail our (approximate) sfermion mass cut. The (golden) diamonds denote points where $\chi$ is not the LSP, and the (blue) squares denote points that survive all the LEP cuts. smaller cross sections, and the under-dense points larger cross sections before applying the rescaling correction. After rescaling, the under-dense points yield cross sections in the range found for the favored points with $0.1 \leqslant \Omega_{\chi} h^{2} \leqslant 0.3$, denoted by (blue) boxes. For $\tan \beta=10$ and $\mu>0$, the scalar cross section is about an order of magnitude higher for points which survive all cuts. Relative to the cases with $\mu>0$, the $\mu<0$ cases have a scalar cross section which is $1-2$ orders of magnitude smaller, because of the $b \rightarrow s \gamma$-motivated cut $m_{A}$ $>300 \mathrm{GeV}$ for $\mu<0$.

A comparison with Fig. 2 shows that the largest cross sections displayed in Figs. 4(a,b), are almost all for Higgsino-like states whose elastic cross section is mediated by $Z$ exchange. These are cosmologically under-dense, due to a combination of large annihilation and coannihilation cross sections. The cosmologically over-dense regions with relatively low elastic cross sections are mainly for gauginolike states, and are for the most part more massive than 300 $\mathrm{GeV}$, which is the oft-quoted upper bound on the bino mass in the MSSM [39].

Our resulting predictions for the spin-dependent elastic neutralino-proton cross section for $\tan \beta=3$ and $\mu>0$, after taking into account the LEP and cosmological constraints, are shown in Fig. 5(a), where a comparison with the UHM case is also made. ${ }^{6}$ The raggedness of the upper and lower boundaries of the dark (blue) shaded allowed region reflect the coarseness of our parameter scan, and the relatively low density of parameter choices that yield cross sections close to these boundaries. We see that, at low $m_{\chi}$ close to the LEP limit, the spin-dependent cross section may be as much as an order of magnitude greater than in the UHM case considered previously [6], shown by the concave (red and turquoise) strip. However, even for low $m_{\chi}$, the attainable range is far below the present experimental sensitivity, which is to $\sigma_{\text {spin }} \sim 1 \mathrm{pb}$, and could be many orders of magnitude lower. As $m_{\chi}$ increases, the maximum allowed value of $\sigma_{\text {spin }}$ decreases, though not as rapidly as in the previous UHM case [6]. The hadronic uncertainties are basically negligible for this spin-dependent cross section, as seen from the light (yellow) shading. Turning now to the option $\tan \beta=3$ and $\mu$ $<0$ shown in Fig. 5(b), we see that the allowed range of the spin-dependent cross section is similar to that in the $\tan \beta$ $=3, \mu>0$ option. This is in contrast to the situation in the UHM [6], where the spin-dependent cross section at low $m_{\chi}$ is much smaller for $\mu<0$ than for $\mu>0$. However, the cross section is still three or more orders of magnitude away from the present experimental upper limit. In the option $\tan \beta$ $=10$ and $\mu>0$ shown in Fig. 5(c), we see that the attainable range of the spin-dependent cross section is again similar to the previous option. This again contrasts with the UHM case, where the narrow allowed band for large $m_{\chi} \sim 500 \mathrm{GeV}$ was somewhat higher than for the option $\tan \beta=3$ and $\mu>0$. As shown in Fig. 5(d), our results for $\tan \beta=10$ and $\mu<0$ are very similar to those for $\mu>0$.

\footnotetext{
${ }^{6}$ In contrast to [6], here we have taken into account the updated LEP constraints.
} 

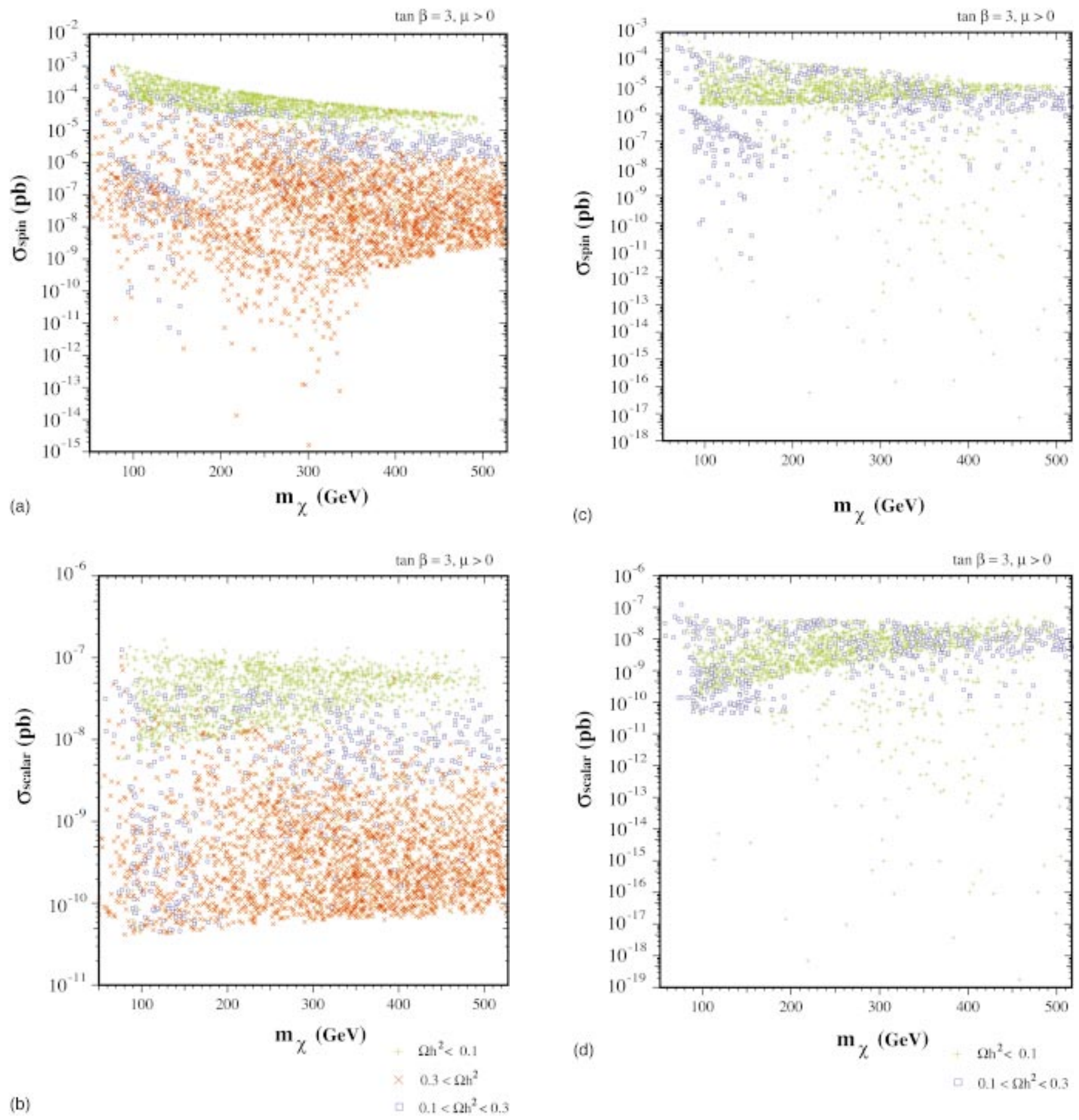

FIG. 4. (Color). Scatter plots (a),(c) of the spin-dependent and (b),(d) of the spin-independent elastic scattering cross sections for $\tan \beta=3, \mu>0$, after implementing the LEP constraints, exhibiting the impacts of the cosmological relic density constraints. The (green) pluses have $\Omega_{\chi} h^{2}<0.1$, the favored (blue) boxes have $0.1 \leqslant \Omega_{\chi} h^{2} \leqslant 0.3$, and the (red) crosses have $\Omega_{\chi} h^{2}>0.3$. Note in panels (c) and (d) the impacts of removing the over-dense points, which tend to have lower cross sections, and rescaling the under-dense points as in Eq. (1), suppressing some high cross section points.

The analogous results for the spin-independent elastic neutralino-proton cross section, after taking into account the LEP and cosmological constraints, are shown in Fig. 6, where comparisons with the UHM case are also made. We see in panel Fig. 6(a) for $\tan \beta=3$ and $\mu>0$ a pattern that is similar to the spin-dependent case. For small $m_{\chi}$, the spinindependent scalar cross section, shown by the dark (blue) shaded region, may be somewhat higher than in the UHM case, shown by the (red and turquoise) diagonal strip, while it could be much smaller. For large $m_{\chi}$, the cross section may be rather larger than in the UHM case, but it is always far below the present sensitivity. The case shown in panel (b) of $\tan \beta=3$ and $\mu<0$ is somewhat different: the cross section never gets to be significantly larger than the UHM value at small $m_{\chi}$. The reason for the anomalous extension of the UHM band outside the more general range is that the newer 

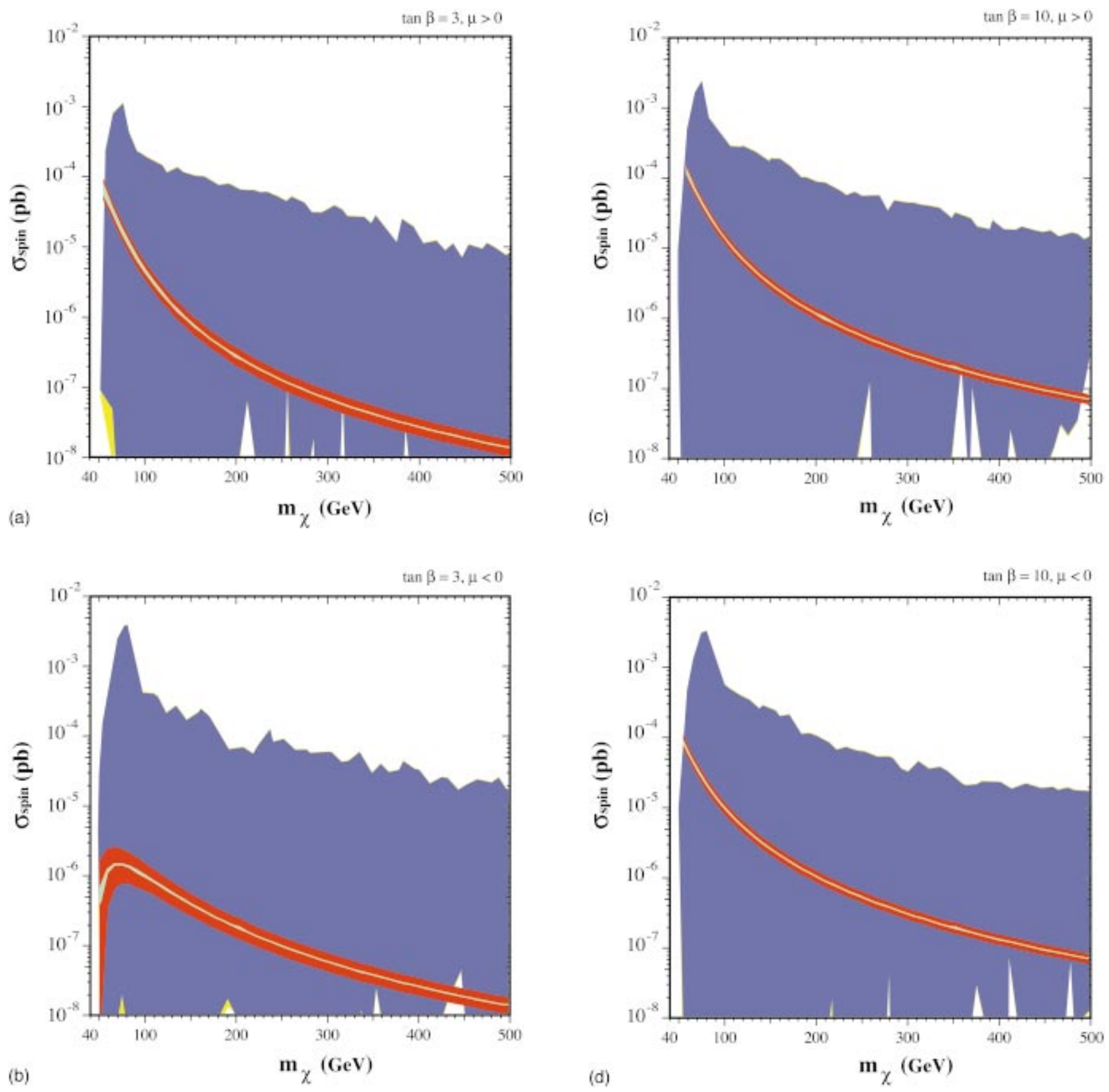

FIG. 5. (Color). Allowed ranges of the spin-dependent elastic neutralino-proton cross section for (a) $\tan \beta=3$ and $\mu>0$, (b) $\tan \beta=3$ and $\mu<0$, (c) $\tan \beta=10$ and $\mu>0$ and (d) $\tan \beta=10$ and $\mu<0$. The main (blue) shaded regions summarize the envelopes of possible values found in our scan, for points respecting the LEP constraints, discarding points with $\Omega_{\chi} h^{2}>0.3$, and rescaling points with $\Omega_{\chi} h^{2}<0.1$ according to Eq. (1). The small light (yellow) shaded extensions of this region reflect the hadronic matrix element uncertainties discussed in Sec. II. The concave (red and turquoise) strips are those found previously assuming universal Higgs scalar masses (UHM) [6].

analysis reflected in the (blue and yellow) shaded region incorporates updated LEP constraints [19], that are significantly stronger for small $\tan \beta$ and small $m_{\chi}$ than those used in [6]. This "anomaly", is absent in panel (c) for $\tan \beta=10$ and $\mu<0$, which closely resembles panel (a), and also panel (d) for $\tan \beta=10$ and $\mu>0$. We note in panel (d) a lesser reappearance of the "anomalous" outdated UHM region at small $m_{\chi}$. The dip in the (red and turquoise) UHM band for $m_{\chi} \sim 230 \mathrm{GeV}$ in panel (d) reflects rather special cancellations [6] that are absent in the more general case. Overall, we note that the hadronic uncertainties, denoted by the light (yellow) bands, are somewhat larger in the spin-independent case than in the spin-dependent case.

\section{SUMMARY AND PROSPECTS}

In this paper we have extended the analysis of [6] to consider a more general sampling of supersymmetric models, relaxing the UHM assumption we made previously. For each of two choices of $\tan \beta$ and $\mu$ negative (positive), we have 

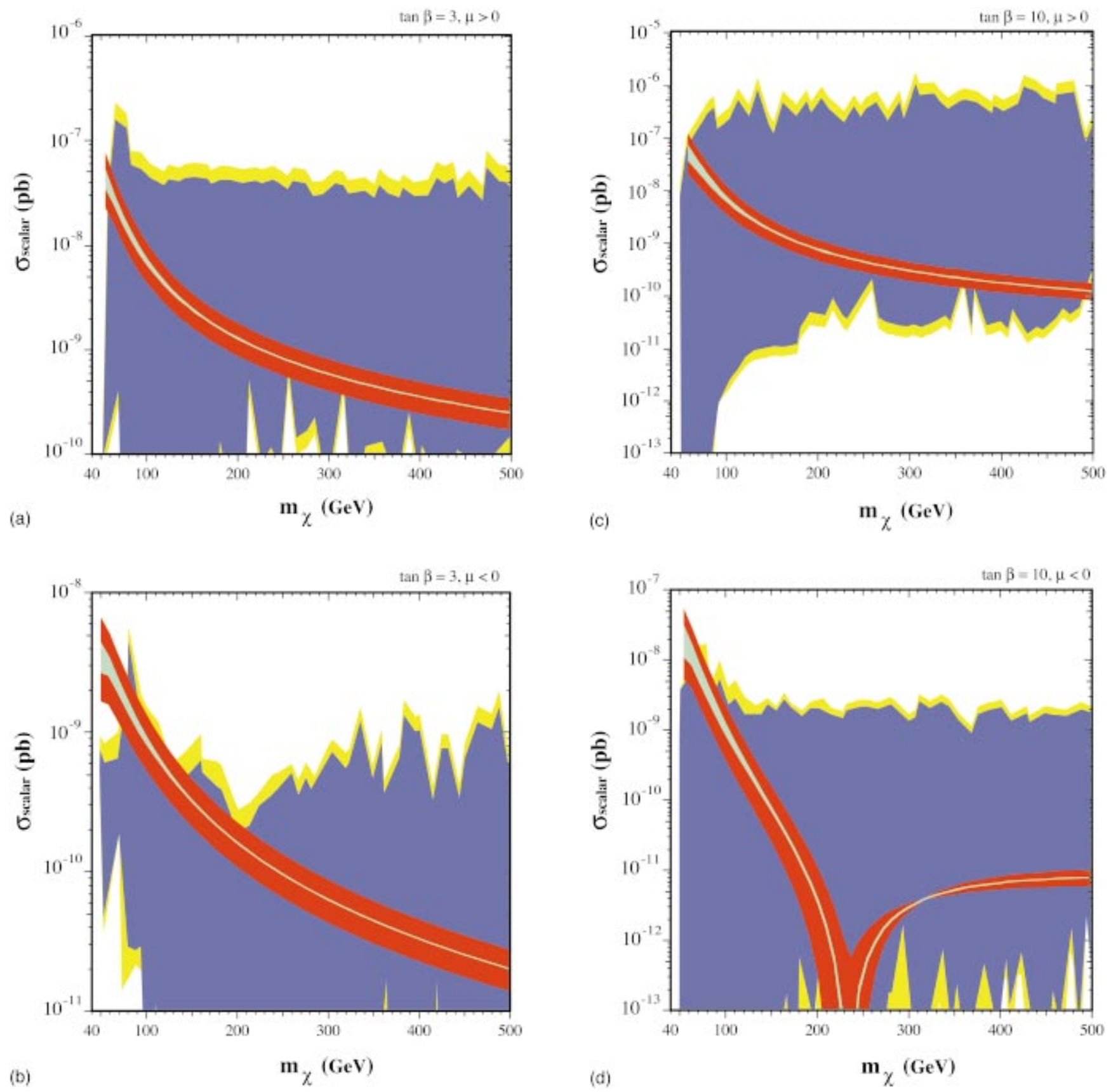

FIG. 6. (Color). Allowed ranges of the spin-independent elastic neutralino-proton cross section for (a) $\tan \beta=3$ and $\mu>0$, (b) $\tan \beta$ $=3$ and $\mu<0$, (c) $\tan \beta=10$ and $\mu>0$ and (d) $\tan \beta=10$ and $\mu<0$. The main (blue) shaded regions summarize the envelopes of possible values found in our scan, for points respecting the LEP constraints, discarding points with $\Omega_{\chi} h^{2}>0.3$, and rescaling points with $\Omega_{\chi} h^{2}$ $<0.1$ according to Eq. (1). The small light (yellow) shaded extensions of this region reflect the hadronic matrix element uncertainties discussed in Sec. II. The (red and turquoise) diagonal strips are the results found assuming universal Higgs scalar masses (UHM) [6].

sampled 70000 (90000) sets of MSSM parameters, 30000 in general scans and 20000 each in two (three) special subscans over lower values of $M_{2}, \mu, m_{0}$ (and $m_{A}$ ). We have implemented the current LEP constraints on MSSM parameters [19], discussing in detail which scan points survive which of these constraints. We have further discussed which of the remaining scan points yield a cosmological relic density in the allowed range $\Omega_{\chi} h^{2} \leqslant 0.3$, and which of these are in the preferred range $\Omega_{\chi} h^{2} \geqslant 0.1$. We exclude from further consideration the over-dense points with $\Omega_{\chi} h^{2}>0.3$, and rescale the predicted cross sections for under-dense points with $\Omega_{\chi} h^{2}<0.1$ as in Eq. (1).

The cross sections we predict for spin-dependent and spin-independent elastic neutralino-proton scattering for different values of $\tan \beta$ and the sign of $\mu$ are shown in Figs. 5 and 6 , respectively. We provide in Fig. 7 a compilation of our results, compared with the present experimental upper limits on the cross sections [13] and the detection of spinindependent scattering reported by the DAMA Collaboration [16]. The light (yellow) shaded regions correspond to the full 

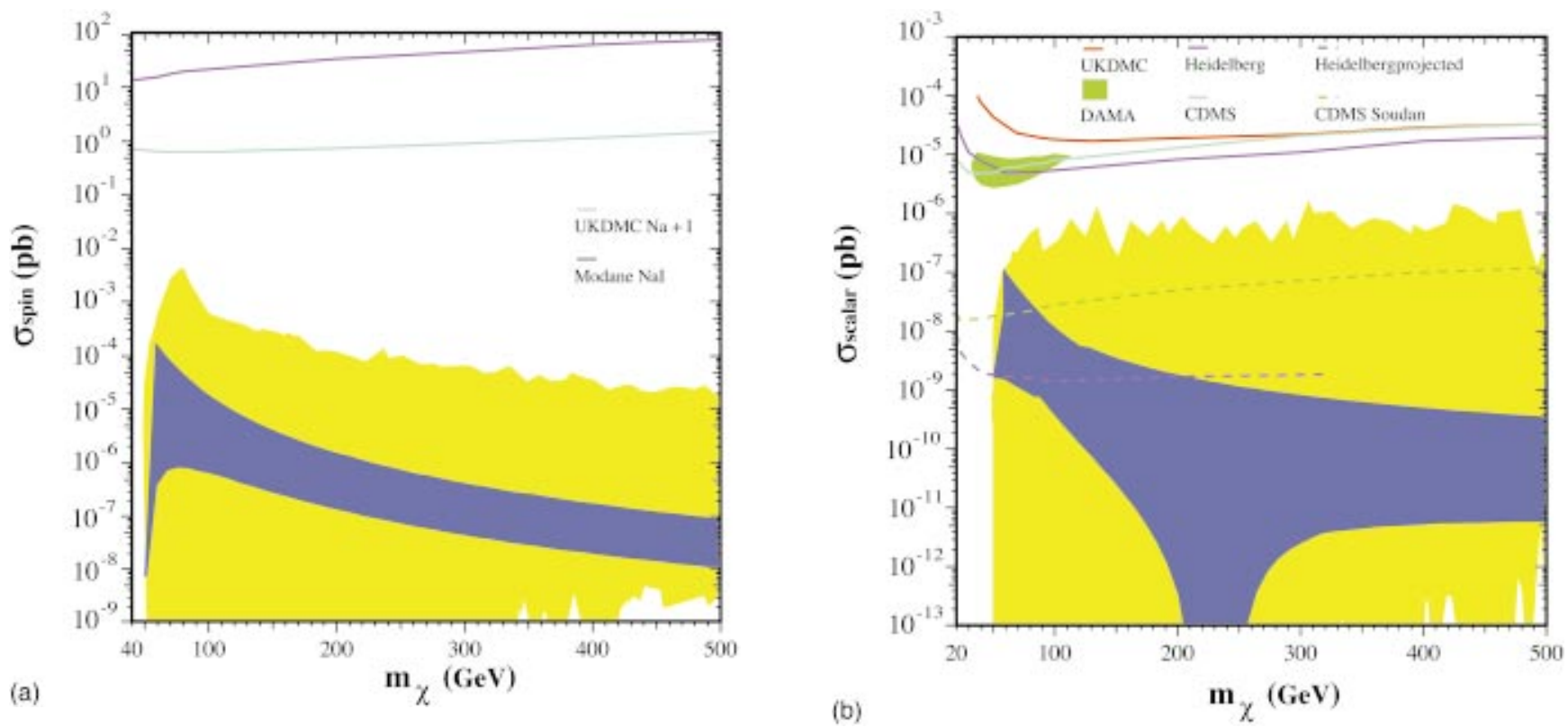

FIG. 7. (Color). Compilations of our allowed ranges for (a) the spin-dependent elastic neutralino-proton cross section, and (b) the spin-independent elastic neutralino-proton cross section for both the values of $\tan \beta$ and the signs of $\mu$ studied. These ranges are shown by the light (yellow) shaded regions. The dark (blue) shaded regions correspond to the allowed ranges when the parameters are restricted by assuming universal Higgs scalar masses (UHM) [6]. Our results are compared in panel (a) with the available experimental upper limits [13], and in panel (b) with the detection reported by the DAMA Collaboration [16], as well as with upper limits from other experiments [13].

range of cross sections we find for the values of $\tan \beta$ and signs of $\mu$ studied. These are contrasted with our previous results [6], when universal Higgs scalar masses (UHM) are assumed, which are shown by the dark (blue) shaded regions in Fig. 7. We see that our predicted cross sections are well below the experimental upper limits for both the spindependent and -independent cases. We are unable to find MSSM parameter sets consistent with our relaxed universality assumptions that come close to explaining the DAMA measurements. Our assumptions would need to be questioned if the neutralino scattering interpretation of the DAMA data is confirmed. We emphasize that we have restricted our attention to models with $\tan \beta \leqslant 10$. We expect that larger values of $\tan \beta$ could lead to somewhat larger cross sections. Alternatively, we emphasize that the DAMA data might favor models with smaller values of $m_{\tilde{q}} / m_{\tilde{l}}$, obtained either by relaxing the input universality assumption, or by imposing it at some renormalization scale below the conventional supersymmetric GUT scale [21].

In the future, we plan to improve the available relic density calculations by extending them to larger $\tan \beta$, incorporating consistently all coannihilation processes, in the context of a general study of MSSM phenomenology at large $\tan \beta$. On the experimental side, we expect that other Collaborations will soon be able to confirm or exclude definitively the DAMA interpretation of their annual modulation signal as being due to neutralino scattering. Looking further ahead, we interpret our results as indicating a high priority for a new generation of direct dark matter detection experiments [40] with a much higher sensitivity.

\section{ACKNOWLEDGMENTS}

We thank Toby Falk and Gerardo Ganis for many related discussions. The work of K.A.O. was supported in part by DOE grant DE-FG02-94ER-40823.
[1] N. Bahcall, J. P. Ostriker, S. Perlmutter, and P. J. Steinhardt, Science 284, 1481 (1999).

[2] For a review, see, e.g., G. Jungman, M. Kamionkowski, and K. Griest, Phys. Rep. 267, 195 (1996).

[3] M. W. Goodman and E. Witten, Phys. Rev. D 31, 3059 (1986).

[4] J. Ellis, J. S. Hagelin, D. V. Nanopoulos, K. A. Olive, and M. Srednicki, Nucl. Phys. B238, 453 (1984).

[5] For reviews, see H. P. Nilles, Phys. Rep. 110, 1 (1984); H. E. Haber and G. L. Kane, ibid. 117, 75 (1995).

[6] J. Ellis, A. Ferstl, and K. A. Olive, Phys. Lett. B 481, 304 (2000).
[7] J. Ellis and R. Flores, Nucl. Phys. B307, 883 (1988); Phys. Lett. B 263, 259 (1991); 300, 175 (1993).

[8] K. Griest, Phys. Rev. D 38, 2357 (1988); R. Barbieri, M. Frigeni, and G. Giudice, Nucl. Phys. B313, 725 (1989); R. Flores, K. A. Olive, and M. Srednicki, Phys. Lett. B 237, 72 (1990); M. Drees and M. M. Nojiri, Phys. Rev. D 48, 3483 (1993); V. Bednyakov, H. V. Klapdor-Kleingrothaus, and S. Kovalenko, ibid. 50, 7128 (1994); H. Baer and M. Brhlik, ibid. 57, 567 (1998).

[9] H. Leutwyler, hep-ph/9609465.

[10] H.-Y. Cheng, Phys. Lett. B 219, 347 (1989). 
[11] J. Gasser, H. Leutwyler, and M. E. Sainio, Phys. Lett. B 253, 252 (1991); M. Knecht, Pin. Newslett. 15, 108 (1999).

[12] G. Mallot, hep-ex/9912040.

[13] R. Gaitskell and V. Mandic, http: //cdms . berkeley.edu/limitplots/.

[14] B. Sadoulet, Nucl. Phys. B (Proc. Suppl.) 77, 389 (1999); A. Bottino, F. Donato, N. Forengo, and S. Scopel, Phys. Rev. D 59, 095003 (1999); 59, 095004 (1999); Astropart. Phys. 10, 203 (1999); 13, 215 (2000); Phys. Rev. D 62, 506006 (2000).

[15] A. Corsetti and P. Nath, hep-ph/0003186.

[16] DAMA Collaboration, R. Bernabei et al., Phys. Lett. B 480, 23 (2000).

[17] CDMS Collaboration, R. Abusaidi et al., Phys. Rev. Lett. 84, 5699 (2000).

[18] J. Ellis, T. Falk, K. A. Olive, and M. Schmitt, Phys. Lett. B 388, 97 (1996); 413, 355 (1997); J. Ellis, T. Falk, G. Ganis, K. A. Olive, and M. Schmitt, Phys. Rev. D 58, 095002 (1998).

[19] J. Ellis, T. Falk, G. Ganis, and K. A. Olive, Phys. Rev. D 62, 075010 (2000).

[20] E. Accomando, R. Arnowitt, B. Dutta, and Y. Santoso, Nucl. Phys. B585, 124 (2000); R. Arnowitt, B. Dutta, and Y. Santoso, hep-ph/0005154.

[21] E. Gabrielli, S. Khalil, C. Munoz, and E. Torrente-Lujan, Phys. Rev. D 63, 025008 (2000).

[22] T. Falk, A. Ferstl, and K. A. Olive, Phys. Rev. D 59, 055009 (1999).

[23] T. Falk, A. Ferstl, and K. A. Olive, Astropart. Phys. 13, 301 (2000).

[24] U. Chattopadhyay, T. Ibrahim, and P. Nath, Phys. Rev. D 60, 063505 (1999).
[25] P. Gondolo and K. Freese, hep-ph/9908390; S. Y. Choi, hep-ph/9908397.

[26] P. de Bernardis et al., Nature (London) 404, 955 (2000); A. E. Lange et al., Phys. Rev. D 63, 042001 (2001).

[27] A. Balbi et al., astro-ph/0005124.

[28] J. Ellis and P. Sikivie, Phys. Lett. B 321, 390 (1994).

[29] J. McDonald, K. A. Olive, and M. Srednicki, Phys. Lett. B B283, 80 (1992).

[30] K. Griest and D. Seckel, Phys. Rev. D 43, 3191 (1991).

[31] S. Mizuta and M. Yamaguchi, Phys. Lett. B B298, 120 (1993).

[32] J. Ellis, T. Falk, and K. A. Olive, Phys. Lett. B 444, 367 (1998); J. Ellis, T. Falk, K. A. Olive, and M. Srednicki, Astropart. Phys. 13, 181 (2000).

[33] C. Boehm, A. Djouadi, and M. Drees, Phys. Rev. D 62, $035012(2000)$

[34] J. L. Feng, K. T. Matchev, and F. Wilczek, Phys. Lett. B 482, 388 (2000).

[35] The latest LEP limits on sparticles and Higgs bosons may be found in links on http://delphiwww.cern. ch/ Offline/physics_links/lepc.html

[36] M. Carena, S. Heinemeyer, C. E. Wagner and G. Weiglein, hep-ph/9912223, and references therein.

[37] P. Nath and R. Arnowitt, Phys. Rev. Lett. 74, 4592 (1995); F. M. Borzumati, M. Drees, and M. M. Nojiri, Phys. Rev. D 51, 341 (1995); H. Baer and M. Brhlik, ibid. 55, 3201 (1997).

[38] H. Baer, M. Brhlik, and D. Castaño, Phys. Rev. D 54, 6944 (1996); S. Abel and T. Falk, Phys. Lett. B B444, 427 (1998).

[39] K. A. Olive and M. Srednicki, Phys. Lett. B 230, 78 (1989); Nucl. Phys. B355, 208 (1991); K. Griest, M. Kamionkowski and M. S. Turner, Phys. Rev. D 41, 3565 (1990).

[40] GENIUS Collaboration, L. Baudis et al., hep-ph/9910205. 